\title{
Personalized Web Search Using Emotional Features
}

\author{
Jianwei Zhang ${ }^{1}$, Katsutoshi Minami², Yukiko Kawai ${ }^{2}$, \\ Yuhki Shiraishi $^{1}$, and Tadahiko Kumamoto ${ }^{3}$ \\ 1 Tsukuba University of Technology \\ 2 Kyoto Sangyo University \\ 3 Chiba Institute of Technology \\ \{zhangjw, yuhkis\}@a.tsukuba-tech.ac.jp, \\ \{i1258131,kawai\}@cc.kyoto-su.ac.jp, \\ kumamoto@net.it-chiba.ac.jp
}

\begin{abstract}
Re-ranking and re-retrieval of search results are useful techniques for satisfying users' search intentions, since current search engines cannot always return user-desired pages at the top ranks. In this paper, we propose a system for personalized Web search considering users' emotional aspects. Given a query topic, the system presents the major emotion tendency on this topic that search results returned from search engines are reflecting. The system also enables users to specify the polarities and strengths of their emotions (e.g., happy or sad, glad or angry, peaceful or strained) on this topic and offers a re-ranking list of initial search results based on the similarity of emotions. Particularly, the system can automatically obtain Web pages with minor emotion tendency on the query topic by extracting sub-queries with opposite emotions and conducting a re-retrieval. Experimental evaluations show the re-ranking and the re-retrieval achieve encouraging search results in comparison with initial search results.
\end{abstract}

\section{Introduction}

Search engines such as Google and Yahoo! have become main tools to obtain information from the Web. They typically provide a list of search results for a user query, ranked by relevancy and popularity. Generally, users only look through Web pages in the top-ranked search results and may often find the information satisfying their search needs. However, current search engines sometimes fail to return user-desired pages at top ranks due to the diversity of users' search intentions. Previous works have been devoted to improving search results in two main directions: (1) re-ranking pages in the initially retrieved list and (2) suggesting query expansion or reformulation for re-retrieving new pages.

Many aspects are utilized to achieve effective re-ranking of initial search results, such as query logs [1], authorship [2], passage centrality [3], social tags [4], re-finding [5], multiple pairwise relationships between pages [6], temporal features [7], and demographical contexts (gender, age and income) [8]. Re-retrieval 
of new pages based on query expansion or reformulation is another effective strategy for improving retrieval accuracy, when initial search results in response to a query contain no pages relevant to users' search intentions. Query expansion or reformulation involves expanding or revising the search query to match additional or new pages by utilizing some technologies and information such as global analysis [9], pseudo-relevance feedback [10], users' personal information repository 11], term classification 12], hints obtained from external Web search engines [13], social annotation [14], wikipedia articles [15], and automatic diagnosis of term mismatch [16].

On the other hand, sentiment analysis and opinion mining [17, 18] have attracted a lot of research interests, which study emotion and its related concepts such as sentiments, opinions and attitudes. The role of emotions in information retrieval is investigated in some researches 19 21. Specially, researches on sentiment retrieval or opinion retrieval [22-27] aim to provide a general opinion search service, similar to traditional Web search in the way that both of them find pages relevant to the query, but different from the latter in the way that sentiment retrieval need further determine whether the pages express opinions on the query topic and whether their polarities are positive or negative.

In this paper, we focus on emotional aspects of users and pages and apply such features to effectively modify search results. Not restricted to positivenegative emotions, we adopt more diverse emotions to the re-ranking and the re-retrieval of search results. Users can not only select three types of emotions ("Happy $\Leftrightarrow$ Sad," "Glad $\Leftrightarrow$ Angry," and "Peaceful $\Leftrightarrow$ Strained."), but also set the strengths of emotions for the re-ranking. These features is also utilized to perform a re-retrieval to obtain pages with opposite emotions. Specially, we propose a system that enables the following:

- construction of emotion dictionary that represents words and their emotion values on three types of emotions.

- extraction of emotion with respect to the query topic, i.e., the major emotion tendency that search results returned from search engines are reflecting.

- interactive re-ranking of initial search results based on the user-specified emotions.

- automatic re-retrieval of new search results reflecting the opposite emotions.

Figure 1 is an example of initial search results for a query topic "Child benefit." Except for Web pages, the system also provides the emotions with respect to the query topic. Users can set their emotions and conduct a re-ranking so as to obtain a list sorted by the similarity between each initial search result's emotions and the user-specified emotions (Figure 2). Moreover, we observe that users tends to set the emotions opposite to the major emotion tendency for obtaining information in multiple perspective. Therefore, we also propose an opposite re-retrieval without user's input for automatically finding pages with minor emotion tendency (Figure 3). The query topic "Child benefit" in this example is a law introduced in Japan about distributing social security payment to the parents of children. The emotions on this topic that initial search results reflect are a little sad, a little angry and a little strained (Figure1), because most 

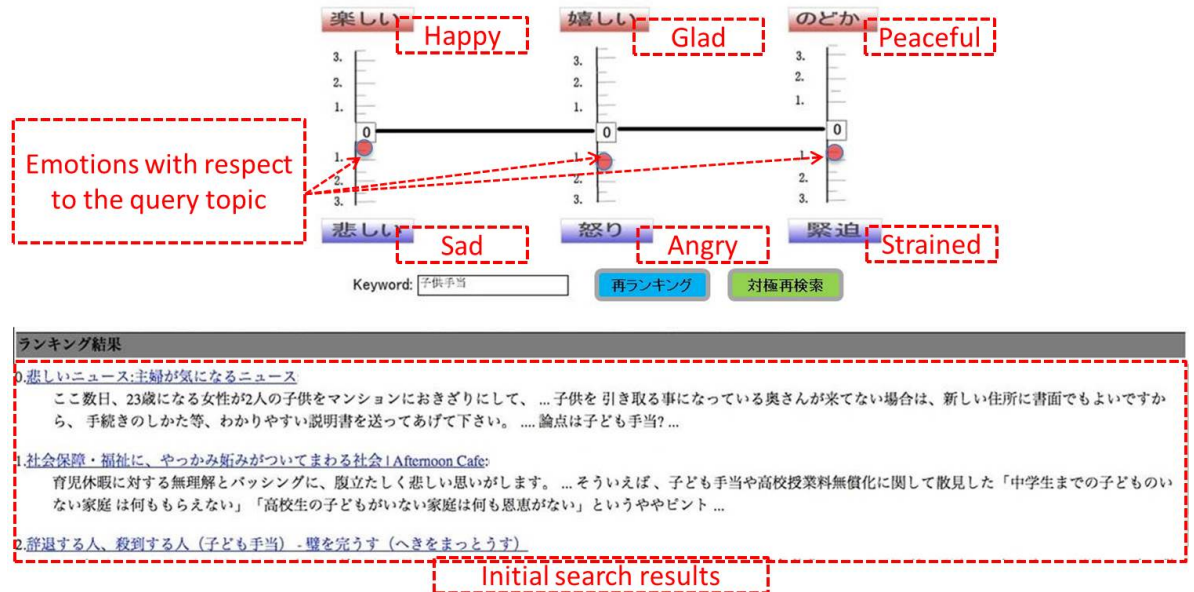

Fig. 1. Emotions with respect to the query topic

(1) Users set their emotions

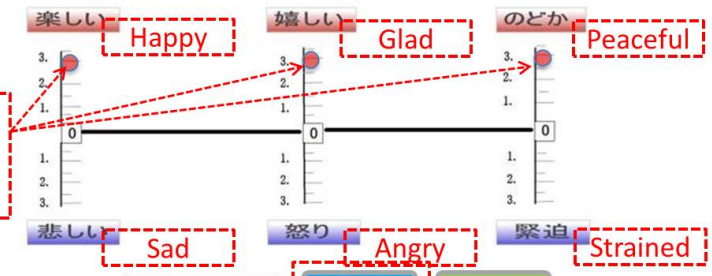

(2) Re-ranking

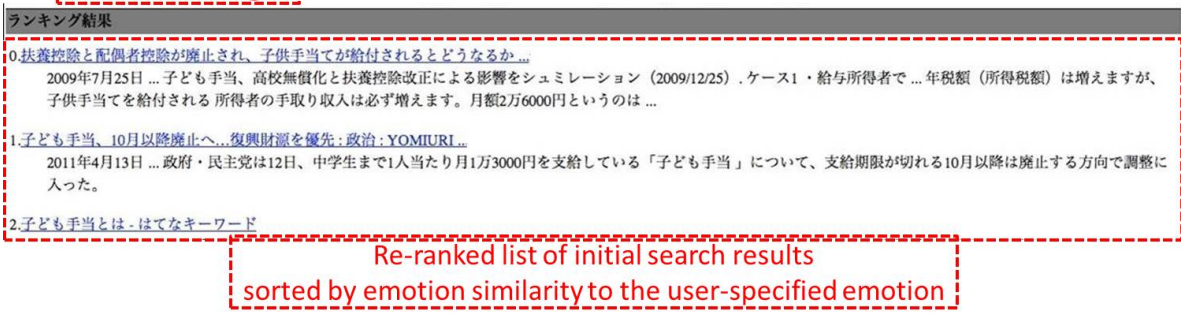

Fig. 2. Re-ranking of initial search results based on the user-specified emotion 


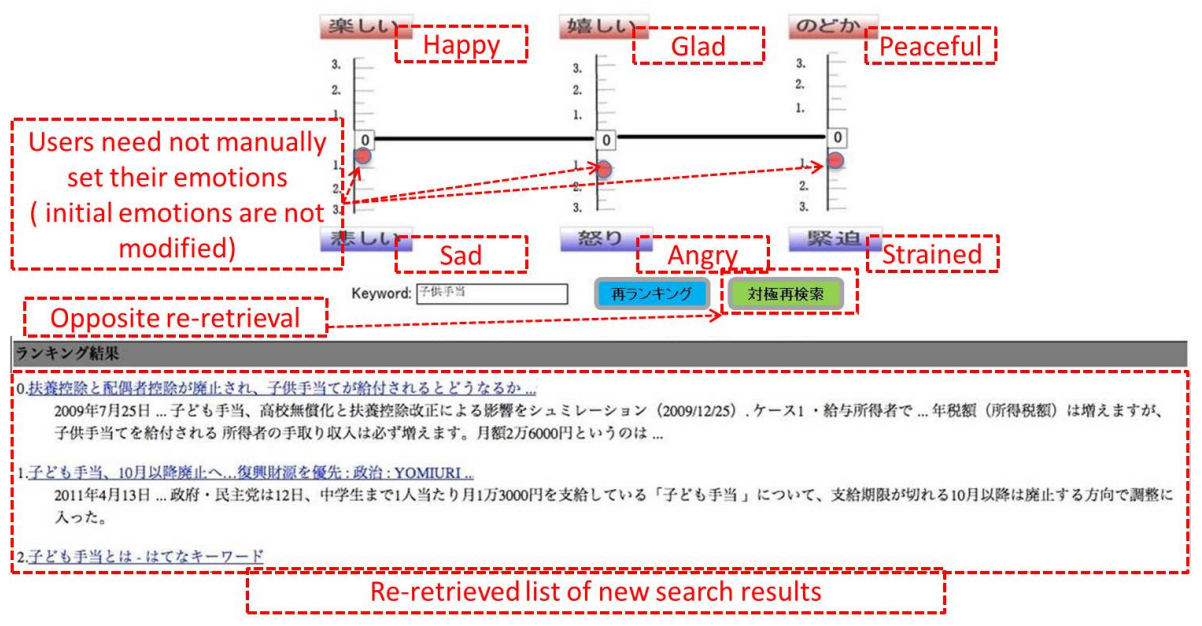

Fig. 3. Re-retrieval of new search results reflecting the opposite emotions

of pages in the initial search list introduce that many people decline this offer, or parents may abuse this grant. When a user sets the opposite emotions and conducts a re-ranking (Figure 2) or conducts an opposite re-retrieval without manually specifying any emotion (Figure 3), the pages with positive emotions can be obtained. For example, some pages at the top ranks of the re-ranking and the re-retrieval lists argue that child benefit is effective in stimulating economic growth and thus express happy, glad and peaceful emotions.

The rest of this paper is structured as follows. Section 2 provides an overview of the system. Section 3 describes the emotion calculation for search results. Section 4 describes how to modify search results using the emotional features. Section 5 evaluates the effectiveness of our system. Section 6 reviews related work. Finally we conclude the paper and discuss future work in Section 7.

\section{System Overview}

Figure 4 shows the overview of the proposed system. Given a query topic from a user, the system performs the following process:

1. Initial search results are returned by using Yahoo! Web Search API [28]. Specially, titles and snippets of pages are obtained for emotion analysis.

2. Emotions with respect to the query topic are calculated. The emotion values for each page ('s title and snippet) are calculated using an emotion dictionary that we have developed, and the averages of emotion values of initial search results are used as the emotions with respect to the query topic, that actually reflect the major emotion tendency of initial search results. The details of emotion calculation are described in Section 3 . 


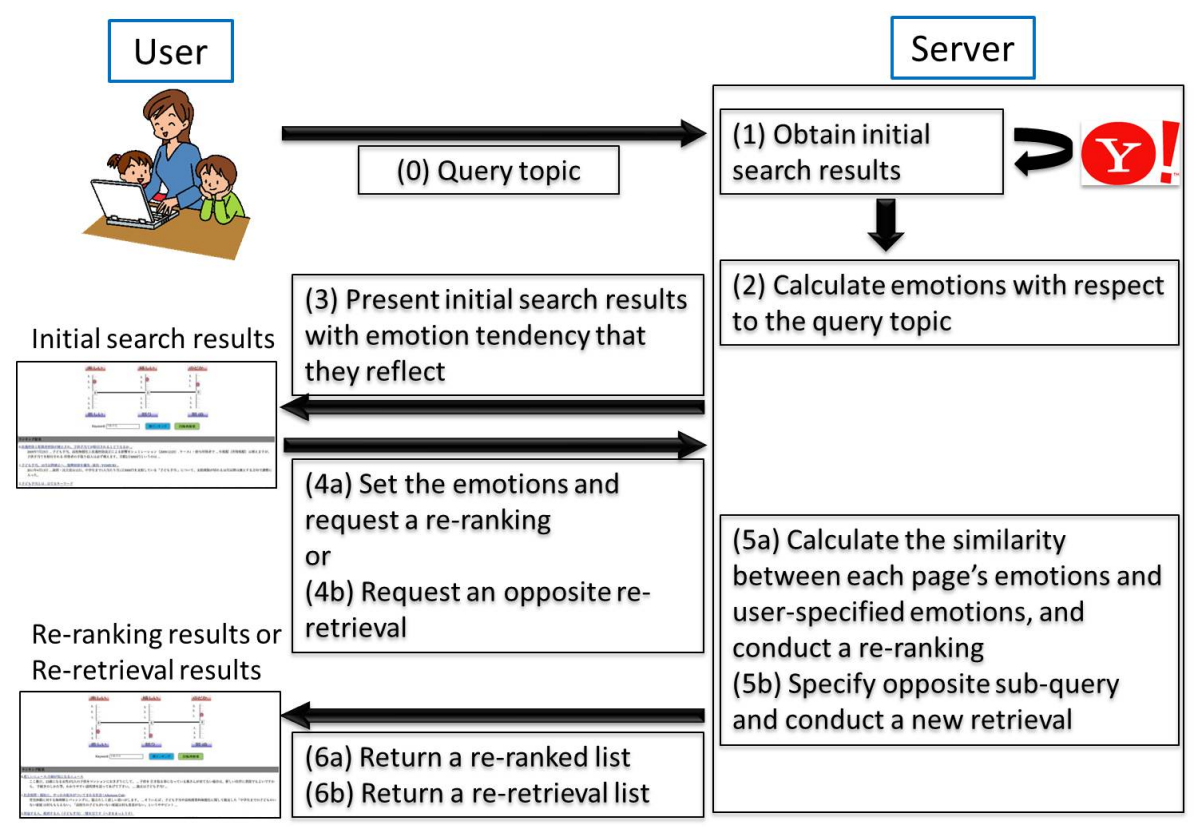

Fig. 4. Overview of the re-ranking and re-retrieval system

3. Both the initial search results and the emotion with respect to the query topic are presented to the user (Figure 1). The emotions have three dimensions and are presented as a graph.

4a. The user can set the emotions by adjusting the strength of emotion on each dimension, and request a re-ranking of initial search results based on emotional features (Figure 2). For example, the initial search results in response to the query topic "Child benefit" reflect that the major emotion tendency is "a little sad," while the user can set stronger emotion "very sad" or opposite emotion "very happy." Re-ranking is expected to sort the pages with emotions close to the user-specified one to the top ranks.

4b. In the case that the user wants to find pages with minor emotion tendency, re-ranking of the initial search results may not satisfy the user's needs if there are not the pages with opposite emotions in the initial search list. Therefore, the system enables the user to alternatively request an opposite re-retrieval (Figure 3). The re-retrieval is expected to find new pages with opposite emotions, not restricted to the initial search results. The user need not manually set the emotions for the opposite re-retrieval.

5a. For the re-ranking, the similarity between each page's emotions and the userspecified emotions is calculated. The re-ranked list of initial search results is sorted in the descending order of emotion similarity. The details of re-ranking are described in Section 4.1 . 
Table 1. A sample of the emotion dictionary

\begin{tabular}{l|c|c|c}
\hline Word $w$ & $s(w)$ on Happy $\Leftrightarrow \operatorname{Sad}$ & $s(w)$ on Glad $\Leftrightarrow$ Angry & $s(w)$ on Peaceful $\Leftrightarrow$ Strained \\
\hline prize & 0.862 & 1.000 & 0.808 \\
cooking & 1.000 & 0.653 & 0.881 \\
deception & 0.245 & 0.075 & 0.297 \\
death & 0.013 & 0.028 & 0.000 \\
\hline
\end{tabular}

Table 2. Original emotion words for the three dimensions

\begin{tabular}{l|l}
\hline Dimension & Original emotion words \\
\hline Happy & Happy, Enjoy, Enjoyment, Joy $\left(O W_{L}\right)$ \\
$\Leftrightarrow$ Sad & Sad, Grieve, Sadness, Sorrow $\left(O W_{R}\right)$ \\
\hline Glad & Glad, Delightful, Delight $\left(O W_{L}\right)$ \\
$\Leftrightarrow$ Angry & Angry, Infuriate, Rage $\left(O W_{R}\right)$ \\
\hline Peaceful & Peaceful, Mild, Primitive, Secure $\left(O W_{L}\right)$ \\
$\Leftrightarrow$ Strained & Tense, Eerie, Worry, Fear $\left(O W_{R}\right)$ \\
\hline
\end{tabular}

5b. For the re-retrieval, the page with opposite emotion is first extracted from the initial search results. Then opposite sub-query extracted from this page expands the initial query to match new pages with opposite emotion. The details of re-retrieval are described in Section 4.2.

6. Finally, the re-ranking results or the re-retrieval results are presented to the user.

\section{Calculation of Emotions with Respect to the Query Topic}

\subsection{Construction of the Emotion Dictionary}

We construct an emotion dictionary, in which each entry indicates the correspondence of a word and its emotion values on three dimensions. The three-dimension emotions are "Happy $\Leftrightarrow$ Sad," "Glad $\Leftrightarrow$ Angry," and "Peaceful $\Leftrightarrow$ Strained," that are formed based on a statistical analysis and a clustering analysis in our previous work [29]. A sample of the emotion dictionary is shown in Table 1, A emotion value $s(w)$ of a word $w$ on each dimension is a value between 0 and 1. The values close to 1 mean the emotions of the words are close to "Happy," "Glad," or "Peaceful," while the values close to 0 mean the words' emotions are close to "Sad," "Angry," or "Strained." For example, the emotion value of the word "prize" on "Happy $\Leftrightarrow$ Sad" is 0.862 , which means the word "prize" conveys a "Happy" emotion. The emotion value of the word "deception" on "Glad $\Leftrightarrow$ Angry" is 0.075, which means "deception" conveys an "Angry" emotion. 
For each of the three dimensions, we set two opposite sets $\left(O W_{L}\right.$ and $\left.O W_{R}\right)$ of original emotion words (Table2). The basic idea of emotion dictionary construction is that a word expressing a left emotion on a dimension often occurs with the dimension's $O W_{L}$, but rarely occurs with its $O W_{R}$. For example, the word "prize" expressing the emotion "Happy" often occurs with the words "Happy," "Enjoy," "Enjoyment," "Joy," but rarely occurs with the words "Sad," "Grieve," "Sadness," "Sorrow." We compare the co-occurrence of each target word with the two sets of original emotion words for each dimension by analyzing the news articles published by a Japanese newspaper YOMIURI ONLINE during 2002 2006.

First, for each dimension, we extract the set $S$ of news articles including one or more original emotion words in $O W_{L}$ or $O W_{R}$. Then, for each news article, we count the numbers of the words that are included in $O W_{L}$ and in $O W_{R}$. The news articles, in which there are more words included in $O W_{L}$ than in $O W_{R}$, constitute the set $S_{L}$. Inversely, the news articles, in which there are more words included in $O W_{R}$ than in $O W_{L}$, constitute the set $S_{R} \cdot N_{L}$ and $N_{R}$ represent the numbers of the news articles in $S_{L}$ and $S_{R}$, respectively. For each word $w$ occurring in the set $S$, we count the number of news articles including $w$ in $S_{L}$ and mark it as $N_{L}(w)$. Similarly, we count and mark the number of news articles including $w$ in $S_{R}$ as $N_{R}(w)$. The conditional probabilities are

$$
P_{L}(w)=\frac{N_{L}(w)}{N_{L}} \quad P_{R}(w)=\frac{N_{R}(w)}{N_{R}}
$$

A emotion value $s(w)$ of a word $w$ is calculated as follows:

$$
s(w)=\frac{P_{L}(w) * \text { weight }_{L}}{P_{L}(w) * \text { weight }_{L}+P_{R}(w) * \text { weight }_{R}}
$$

where weight $_{L}=\log _{10} N_{L}$ and weight $_{R}=\log _{10} N_{R}$.

\subsection{Emotion Calculation for Individual Pages and Emotion Summary of Search Results}

The emotion values of an individual page are calculated by looking up the emotion values of the words in the page from the emotion dictionary and averaging them 1 . In this way, a page has an emotion value ranging from 0 to 1 , since the emotion values of the words in the emotion dictionary range from 0 to 1 . Considering the comprehensibility and the symmetry, the emotion value $(\mathrm{x})$ of a page is further converted to a value (y) ranging from -3 to 3 by the formula: $(y=6 * x-3)$. When $\mathrm{x}$ is $1,0.5$, and 0 , the corresponding $\mathrm{y}$ becomes 3,0 , and -3 . The emotion values $3,2,1,0,-1,-2,-3$ on a dimension, e.g., "Happy $\Leftrightarrow$ Sad," correspond to "Happy," "Relatively happy," "A little happy," "Neutral," "A little sad," "Relatively sad" and "Sad" respectively.

\footnotetext{
${ }^{1}$ Since the title and snippet of a page summarize the content of the page and their text is shorter than full page, the system actually calculate the emotion values using the text of the title and snippet for each page so as to shorten the response time.
} 
Emotion values on each dimension of search results in response to a query are averaged as the emotion (polarity and strength) on that dimension with respect to the query. As shown in Figure 1, Figure 2, and Figure 3, the graph has opposite emotion polarities for the three dimensions, thus represents the emotion strengths as their absolute values, although the values of negative emotions in the inner system are negative numbers.

\section{Modification of Search Results Based on Emotional Features}

\subsection{Re-ranking of Initial Search Results Based on User-Specified Emotions}

After receiving the three emotion values specified by the user, the system generates an emotion query vector $V_{q}=\left(v_{q 1}, v_{q 2}, v_{q 3}\right)$ using the emotion value on each dimension as its element. For each page in the initial search results list, an emotion page vector $V_{p}=\left(v_{p 1}, v_{p 2}, v_{p 3}\right)(\mathrm{p}=1, \ldots, \mathrm{N}$, where $\mathrm{N}$ is the number of initial search results) is determined, the elements of which are the emotion values on three dimensions of each page. The similarity between $V_{q}$ and $V_{p}$ is calculated using the measure of cosine similarity:

$$
\operatorname{sim}\left(V_{q}, V_{p}\right)=\frac{v_{q 1} v_{p 1}+v_{q 2} v_{p 2}+v_{q 3} v_{p 3}}{\sqrt{v_{q 1}^{2}+v_{q 2}^{2}+v_{q 3}^{2}} \times \sqrt{v_{p 1}^{2}+v_{p 2}^{2}+v_{p 3}^{2}}}
$$

The initial search results are re-ranked in a descending order of the emotion similarity and presented to the user. The pages with emotions similar to the user-specified ones tend to be ranked to the top place.

\subsection{Re-retrieval of New Search Results Reflecting Opposite Emotions}

The essential of the re-retrieval is to extract the sub-query to expand the initial query. For obtaining pages reflecting opposite emotions, we extract the opposite sub-query as follows:

1. The emotions on the three dimensions with respect to the initial query (emotion summary of initial search results) are represented as an emotion query vector $V_{q}=\left(v_{q 1}, v_{q 2}, v_{q 3}\right)$. Each page in the initial search results list is also represented as an emotion page vector $V_{p}=\left(v_{p 1}, v_{p 2}, v_{p 3}\right)$. The system compares the emotion polarity on each dimension between $V_{q}$ and $V_{p}$, and determines the candidate opposite pages if emotion polarities on all of the three dimensions of a page are contrary to the emotion query.

2. The system then calculates the Euclidean distance $\sqrt{\sum_{i=1}^{3}\left(v_{q i}-v_{p i}\right)^{2}}$ between $V_{q}$ and $V_{p}$ of each candidate opposite page. The page with the largest distance is determined as the opposite page. 
Table 3. Re-ranking effect

\begin{tabular}{c|c|c|c|c}
\hline Query topics & Emotion dimension & $\begin{array}{c}\text { Emotion value } \\
\text { of initial search results }\end{array}$ & $\begin{array}{c}\text { User-specified } \\
\text { emotion value }\end{array}$ & $\begin{array}{c}\text { Emotion value } \\
\text { after re-ranking }\end{array}$ \\
\hline Child benefit & Happy $\Leftrightarrow$ Sad & -0.33 & 0 & 0 \\
& Glad $\Leftrightarrow$ Angry & -1.06 & 3 & 3 \\
& Peaceful $\Leftrightarrow$ Strained & -0.77 & 3 & 3 \\
\hline Citizen judge & Happy $\Leftrightarrow$ Sad & -0.61 & 3 & 3 \\
& Glad $\Leftrightarrow$ Angry & -0.81 & 3 & 3 \\
& Peaceful $\Leftrightarrow$ Strained & -0.63 & 3 & 3 \\
\hline
\end{tabular}

3. Keywords are extracted from the opposite page by using Yahoo! Term Extraction API [30]. The keywords whose scores are larger than 40 are determined as the candidate sub-queries.

4. The system looks up the emotion value of each candidate sub-query from the emotion dictionary, converts the emotion values to the scale ranging from -3 to 3 , and forms an emotion sub-query vector $V_{s}=\left(v_{s 1}, v_{s 2}, v_{s 3}\right)$. Then the system compares the Euclidean distance between $V_{q}$ and each $V_{s}$. The keyword with the largest distance is determined as the opposite sub-query, and is utilized to expand the initial query for the re-retrieval.

\section{Experimental Evaluation}

\subsection{Re-ranking Effect}

We select two query topics for initial retrieval, modify the emotions of initial search results, and then conduct a re-ranking as described in Section 4.1, Table 3 shows the emotions of initial search results, the user-specified emotions, and the emotions after re-ranking. The emotion value on each dimension of initial search results is the average of emotion values of initial search results. The emotion value on each dimension after re-ranking is determined by averaging five students' evaluations on the top ten re-ranked pages. Each student read each top-ranked page and gave a level from -3 to 3 about the emotion polarity and strength that the page reflected.

From Table 3, we observe that if users set emotions different from the major emotion tendency of initial search results, the emotions after re-ranking are consistent with the user-specified emotions. This indicates that the re-ranking based on emotional features can bring the pages with emotions similar to the user-specified emotions to the top ranks.

In the experiments, we observe that users tend to set opposite emotions so as to browse different opinions. However, if there are no pages with opposite emotions in the initial search results list, it is impossible to find them by reranking. In that case expanding the initial query and conducting a re-retrieval are necessary for obtaining such pages. In the next section, we describe the re-retrieval effect. 
Table 4. Query topics and opposite sub-queries (translated from Japanese)

\begin{tabular}{c|c|c}
\hline Query ID & Positive Query & Opposite sub-query \\
\hline$(1)$ & Melting pot & Mixed breed \\
$(2)$ & The University of Tokyo & Consumption \\
$(3)$ & Internet cafe & Dispatch \\
$(4)$ & April fool & Self-restraint \\
\hline Query ID & Negative Query & Opposite sub-query \\
\hline$(5)$ & Press conference & Campaign \\
$(6)$ & Hashimoto Toru & Schedule \\
$(7)$ & Citizen judge & Plan \\
$(8)$ & Tokyo Electric Power Company & Energy \\
\hline Query ID & Mixed Query & Opposite sub-query \\
\hline$(9)$ & Old age & Strength \\
$(10)$ & Job hunting & Media \\
$(11)$ & Bubble economy & Work \\
$(12)$ & Security camera & Particular \\
\hline
\end{tabular}

\subsection{Re-retrieval Effect}

We select twelve query topics for verifying the effect of the opposite re-retrieval described in Section 4.2. We compare the emotions with respect to the initial search results with the emotions with respect to the re-retrieved search results. The twelve query topics are categorized into three types based on their emotions with respect to the initial search results. If the emotions on all of the three dimensions of the initial search results are positive (Happy, Glad, and Peaceful), the query topic is categorized into Positive Query. If the emotions on all of the three dimensions of the initial search results are negative (Sad, Angry, and Strained), the query topic is categorized into Negative Query. If both positive and negative dimension exist (e.g., Happy, Glad, and Strained, etc.), the query topic is categorized into Mixed Query. Table 4 shows the query topics and their extracted opposite sub-queries.

Figure [5, Figure 6] and Figure 7 show the comparison results corresponding to each emotion dimension, respectively for positive queries, negative queries and mixed queries. The abscissa in each graph is query topics and the ordinate is emotion values. The left white bars represent the emotions with respect to the initial search results, and the right black bars represent the emotions with respect to the opposite re-retrieval search results.

From Figure 5, we can observe that for all the positive query topics, the emotion values become smaller or even change to opposite polarities. The overall average of emotion values for all the four positive query topics and all the three dimensions inclines to the negative side in a scale of 0.64 . For the negative query topics in Figure 6] all except Query 7 (Citizen judge) on the dimension "Peaceful $\Leftrightarrow$ Strained" obtain the emotions inclining to the positive side. The overall incline scale from the negative side to the positive side is 0.54 . Opposite re-retrieval is also effective for the mixed query topics in Figure 7 Except Query 
11 (Bubble economy) on the dimensions "Happy $\Leftrightarrow$ Sad" and "Glad $\Leftrightarrow$ Angry," opposite re-retrieval succeeds to obtain search results with emotions inclining to the reverse side for all other mixed query topics. The overall incline scale (from negative to positive, or from positive to negative) for the mixed query topics is 0.14 .

There are some comprehensible pages for comparing the contents of the initial and re-retrieved search results. For example, for Query 4 (April fool), the initial search results contain the pages that introduce tricks for April fool and express happy emotions, while the pages in the top re-retrieved search results are the sad pages about refraining from tricks. Another comprehensible example is Query 8 (Tokyo Electric Power Company). The pages in the initial search list in response to this query are the negative pages expressing considerable criticism to the way that TEPCO handled the crisis of nuclear accidents, while opposite reretrieval brings the positive pages that introduce TEPCO's efforts to develop future energy.

\section{Related Work}

There have been a number of studies on re-ranking search results considering various aspects. Zhuang et al. 1] proposed a Q-Rank method to refine the ranking of search results by constructing the query context from query logs. Bogers et al. [2] utilized the authorship information to extract expert rankings. Bendersky et al. 3] presented a passage-based approach to leverage information about the centrality of the document passages with respect to the initial search results list. Yan et al. 4] proposed a Query-Tag-Gap algorithm to re-rank search results based on the gap between search queries and social tags. Tyler et al. [5] utilized the prediction of re-finding (finding the pages that users have previously visited) to re-rank pages. Kang et al. [6] proposed to combine multiple pairwise relationships between documents to re-rank search results. Chang et al. 7] exploited temporal features for re-ranking time-sensitive search results. Kharitonov et al. [8] improved search results by using demographical contexts such as gender, age, and income.

Another research direction for improving retrieval accuracy is to re-retrieve new search results based on query expansion or reformulation. Xu et al. [9] showed that using global analysis such as word context and phrase structure on local documents (the documents retrieved by the initial query) produced more effective search results. Tao et al. 10] proposed to integrate the initial query with pseudo-relevance feedback documents in a probabilistic mixture model without parameter tuning. Chirita et al. [11] expanded short queries by analyzing user data at different levels ranging from term and compound level analysis up to global co-occurrence statistics. Cao et al. [12] argued against the assumption of pseudo-relevance feedback that the most frequent terms in the pseudo-feedback documents are useful for the retrieval, and proposed to integrate a term classification process to predict the usefulness of expansion terms. Yin et al. [13] utilized the hints such as query logs, snippets and search result documents from 


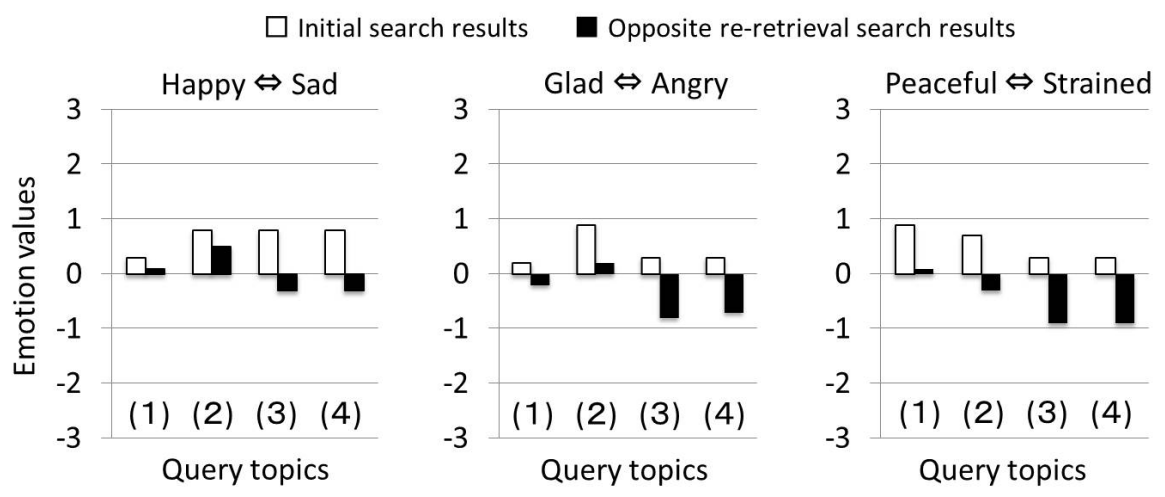

Fig. 5. Positive query

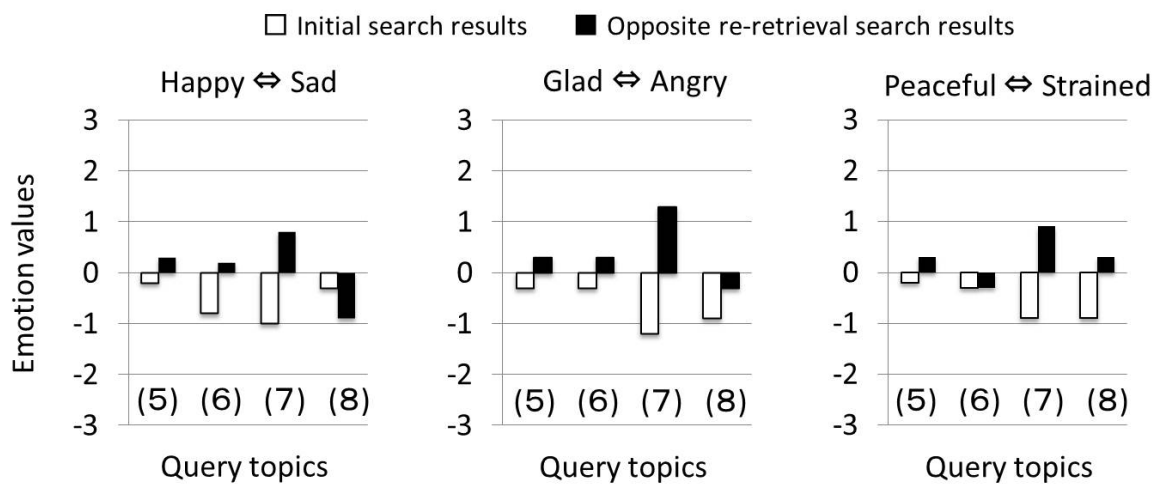

Fig. 6. Negative query

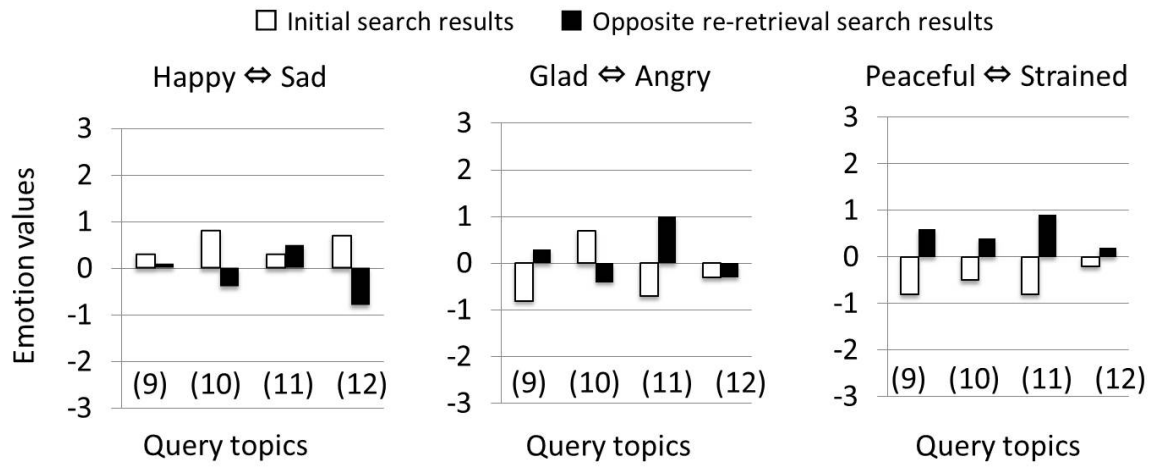

Fig. 7. Mixed query 
external search engines to expand the initial query. Lin et al. [14] extracted candidate expansion terms by a term-dependency method and ranked them based on social annotation resource. Oliveira et al. [15] proposed to expand entityrelated queries using wikipedia articles and tag recommendation methods. Zhao et al. 16] proposed an automatic diagnosis of term mismatch to guide interactive query expansion or create conjunctive queries.

Similar to these researches we also aim to obtain a user-desired re-ranking and re-retrieval search results. Different from them we improve a personalized Web search using the emotional features. The pages with emotions similar to users' can be re-ranked to the top rank and the pages with opposite emotions can be re-retrieved based on the extraction of opposite sub-queries.

On the other hand, sentiment analysis and opinion mining [17, 18] are one of the hottest research areas that extract sentiments (or emotion, opinions, attitudes) from text such as movie reviews, book reviews, and product evaluations. Some researches have applied emotion knowledge to information retrieval and its relevant research areas. Arapakis et al. [19] found that emotions in the information seeking process interwove with physiological, psychological and cognitive processes and formed characterized patterns according to specific task and specific user. In [20], Arapakis et al. personalized affective models using facial expression data and compared their performance to a general model for predicting topical relevance. Moshfeghi et al. 21] investigated the role of emotional features in collaborative recommendation and their experimental results showed the emotional features extracted from movie reviews were capable of enhancing recommendation effectiveness.

Specially, sentiment retrieval or opinion retrieval is a newly developed research subject, which requires documents to be retrieved and ranked according the opinions about a query topic. Eguchi et al. 22] proposed several sentiment retrieval models based on probabilistic language models, assuming that users both input query topics and specify sentiment polarity. Similar methods proposed in [23] and 24] unified topic relevance and opinion relevance respectively based on a quadratic combination and a linear combination. A different method proposed by $\mathrm{Li}$ et al. 25] combined topic-sentiment word pairs in a bipartite graph to effectively rank the documents. Opinion retrieval from UGC (User Generated Content) such as blogs [26] and Twitter 27] also yields comparable retrieval performance.

Different the above researches mainly focusing on review documents and positive-negative emotions, we consider any Web pages and more diverse emotions. As we showed in the experiments, there are Web pages that express both positive emotion and negative emotion in different dimensions.

\section{Conclusions and Future Work}

In this paper, we studied the problem of personalized Web search considering emotional aspects: re-ranking initial search results based on user-specified emotions, and re-retrieving new search results with opposite emotions based on 
the extraction of opposite sub-query. Given a query topic, the system shows the major emotion tendency that search results reflect. After users specify their emotions, the search results similar to users' emotions can be re-ranked to the top place. Even without users' interaction, the system also automatically extracts opposite sub-query so as to re-retrieve the pages with minor emotion tendency with respect to the initial query. We have showed that our system is effective in re-ranking the initial search list and re-retrieving opposite pages.

In the future, we plan to repeat the re-retrieval process and verify whether Web pages with stronger opposite emotions can be extracted. In that case, the problems about the reversion of emotion polarity (from positive to negative, then back to positive) and the topic drift must be taken into consideration.

Acknowledgments. This work was supported by JSPS KAKENHI Grant Number 24800007, 23500140, 24500134, 24780248.

\section{References}

1. Zhuang, Z., Cucerzan, S.: Re-ranking search results using query logs. In: CIKM 2006, pp. 860-861 (2006)

2. Bogers, T., van den Bosch, A.: Authoritative re-ranking of search results. In: Lalmas, M., MacFarlane, A., Rüger, S.M., Tombros, A., Tsikrika, T., Yavlinsky, A. (eds.) ECIR 2006. LNCS, vol. 3936, pp. 519-522. Springer, Heidelberg (2006)

3. Bendersky, M., Kurland, O.: Re-ranking search results using document-passage graphs. In: SIGIR 2008, pp. 853-854 (2008)

4. Yan, J., Liu, N., Chang, E.Q., Ji, L., Chen, Z.: Search result re-ranking based on gap between search queries and social tags. In: WWW 2009, pp. 1197-1198 (2009)

5. Tyler, S.K., Wang, J., Zhang, Y.: Utilizing re-finding for personalized information retrieval. In: CIKM 2010, pp. 1469-1472 (2010)

6. Kang, C., Wang, X., Chen, J., Liao, C., Chang, Y., Tseng, B.L., Zheng, Z.: Learning to re-rank Web search results with multiple pairwise features. In: WSDM 2011, pp. 735-744 (2011)

7. Chang, P., Huang, Y., Yang, C., Lin, S., Cheng, P.: Learning-based time-sensitive re-ranking for Web search. In: SIGIR 2012, pp. 1101-1102 (2012)

8. Kharitonov, E., Serdyukov, P.: Demographic context in Web search re-ranking. In: CIKM 2012, pp. 2555-2558 (2012)

9. Xu, J., Croft, W.B.: Query expansion using local and global document analysis. In: SIGIR 1996, pp. 4-11 (1996)

10. Tao, T., Zhai, C.: Regularized estimation of mixture models for robust pseudorelevance feedback. In: SIGIR 2006, pp. 162-169 (2006)

11. Chirita, P., Firan, C.S., Nejdl, W.: Personalized query expansion for the Web. In: SIGIR 2007, pp. 7-14 (2007)

12. Cao, G., Nie, J., Gao, J., Robertson, S.: Selecting good expansion terms for pseudorelevance feedback. In: SIGIR 2008, pp. 243-250 (2008)

13. Yin, Z., Shokouhi, M., Craswell, N.: Query expansion using external evidence. In: Boughanem, M., Berrut, C., Mothe, J., Soule-Dupuy, C. (eds.) ECIR 2009. LNCS, vol. 5478, pp. 362-374. Springer, Heidelberg (2009)

14. Lin, Y., Lin, H., Jin, S., Ye, Z.: Social annotation in query expansion: a machine learning approach. In: SIGIR 2011, pp. 405-414 (2011) 
15. Oliveira, V., Gomes, G., Belem, F., Brandao, W.C., Almeida, J.M., Ziviani, N., Goncalves, M.A.: Automatic query expansion based on tag recommendation. In: CIKM 2012, pp. 1985-1989 (2012)

16. Zhao, L., Callan, J.: Automatic term mismatch diagnosis for selective query expansion. In: SIGIR 2012, pp. 515-524 (2012)

17. Pang, B., Lee, L.: Opinion Mining and Sentiment Analysis. Foundations and Trends in Information Retrieval 2(1-2), 1-135 (2007)

18. liu, B.: Sentiment Analysis and Opinion Mining. Morgan \& Claypool Publishers (2012)

19. Arapakis, I., Jose, J.M., Gray, P.D.: Affective feedback: an investigation into the role of emotions in the information seeking process. In: SIGIR 2008, pp. 395-402 (2008)

20. Arapakis, I., Athanasakos, K., Jose, J.M.: A comparison of general vs personalised affective models for the prediction of topical relevance. In: SIGIR 2010, pp. 371-378 (2010)

21. Moshfeghi, Y., Jose, J.M.: Role of emotional features in collaborative recommendation. In: Clough, P., Foley, C., Gurrin, C., Jones, G.J.F., Kraaij, W., Lee, H., Mudoch, V. (eds.) ECIR 2011. LNCS, vol. 6611, pp. 738-742. Springer, Heidelberg (2011)

22. Eguchi, K., Lavrenko, V.: Sentiment retrieval using generative models. In: EMNLP 2006, pp. 345-354 (2006)

23. Zhang, M., Ye, X.: A generation model to unify topic relevance and lexicon-based sentiment for opinion retrieval. In: SIGIR 2008, pp. 411-418 (2008)

24. Huang, X., Croft, W.B.: A unified relevance model for opinion retrieval. In: CIKM 2009, pp. 947-956 (2009)

25. Li, B., Zhou, L., Feng, S., Wong, K.: A unified graph model for sentence-Based opinion retrieval. In: ACL 2010, pp. 1367-1375 (2010)

26. Zhang, W., Yu, C.T., Meng, W.: Opinion retrieval from blogs. In: CIKM 2007, pp. 831-840 (2007)

27. Luo, Z., Osborne, M., Wang, T.: Opinion retrieval in Twitter. In: ICWSM (2012)

28. Yahoo! Web Search API, http://developer.yahoo.co.jp/webapi/search/websearch/v2/websearch.html

29. Zhang, J., Kawai, Y., Kumamoto, T., Nakajima, S., Shiraishi, Y.: Diverse sentiment comparison of news websites over time. In: Jezic, G., Kusek, M., Nguyen, N.-T., Howlett, R.J., Jain, L.C. (eds.) KES-AMSTA 2012. LNCS, vol. 7327, pp. 434-443. Springer, Heidelberg (2012)

30. Yahoo! Term Extraction, http://developer.yahoo.com/search/content/V1/termExtraction.html 